\title{
A Multicentre Pilot Study of a Two-Tier Newborn Sickle Cell Disease Screening Procedure with a First Tier Based on a Fully Automated MALDI-TOF MS Platform
}

\author{
Pierre Naubourg ${ }^{1}$, Marven El Osta ${ }^{1}$, David Rageot ${ }^{2}$, Olivier Grunewald ${ }^{3}$, Gilles Renom ${ }^{3}$, \\ Patrick Ducoroy ${ }^{1}$ and Jean-Marc Périni ${ }^{3, *(1)}$ \\ 1 Biomaneo, 22B boulevard Winston Churchill, F-21000 Dijon, France; pierre.naubourg@biomaneo.com (P.N.); \\ marven.elosta@biomaneo.com (M.E.O.); patrick.ducoroy@biomaneo.com (P.D.) \\ 2 CLIPP, Clinical Innovation Proteomic Platform, Université de Bourgogne Franche Comté, \\ F-21000 Dijon, France; david.rageot@clipproteomic.fr \\ 3 Newborn Screening Laboratory, Biology and Pathology Center, Lille University Medical Centre, \\ F-59000 Lille, France; olivier.grunewald@chru-lille.fr (O.G.); gilles.renom@chru-lille.fr (G.R.) \\ * Correspondence: jean-marc.perini@chru-lille.fr
}

Received: 4 December 2018; Accepted: 21 January 2019; Published: 23 January 2019

\begin{abstract}
The reference methods used for sickle cell disease (SCD) screening usually include two analytical steps: a first tier for differentiating haemoglobin $\mathrm{S}(\mathrm{HbS})$ heterozygotes, $\mathrm{HbS}$ homozygotes and $\beta$-thalassemia from other samples, and a confirmatory second tier. Here, we evaluated a first-tier approach based on a fully automated matrix-assisted laser desorption/ionization time-of-flight mass spectrometry (MALDI-TOF MS) platform with automated sample processing, a laboratory information management system and NeoSickle ${ }^{\circledR}$ software for automatic data interpretation. A total of 6701 samples (with high proportions of phenotypes homozygous (FS) or heterozygous (FAS) for the inherited genes for sickle haemoglobin and samples from premature newborns) were screened. The NeoSickle ${ }^{\circledR}$ software correctly classified $98.8 \%$ of the samples. This specific blood sample collection was enriched in qualified difficult samples (premature newborns, FAS samples, late and very late samples, etc.). In this study, the sensitivity of FS sample detection was found to be $100 \%$ on the Lille MS facility and 99\% on the Dijon MS facility, and the specificity of FS sample detection was found to be $100 \%$ on both MS facilities. The MALDI-MS platform appears to be a robust solution for first-tier use to detect the $\mathrm{HbS}$ variant: it is reproducible and sensitive, it has the power to analyze 600-1000 samples per day and it can reduce the unit cost of testing thanks to maximal automation, minimal intervention by the medical team and good overall practicability. The MALDI-MS approach meets today's criteria for the large-scale, cost-effective screening of newborns, children and adults.
\end{abstract}

Keywords: newborn screening; sickle cell disease; MALDI-TOF; mass spectrometry; thalassemia; prevention

\section{Introduction}

The use of mass spectrometry (MS) to screen newborns for haemoglobinopathies differs from the MS-based diagnosis of a broad range of clinically significant haemoglobin $(\mathrm{Hb})$ variants. Newborn screening requires a robust, high-throughput and cost-effective MS method that solely detects the biomarkers of sickle cell disease (SCD) and $\beta$-thalassemia major. In contrast, the broader diagnostic procedure involves the detection of as many changes in globin proteins as possible in a single step. Newborn screening programs are typically organised as single-tier or two-tier procedures. 
In the two-tier procedure, the first tier is typically a routine MS method (electrospray ionization (ESI)-MS [1-3] or matrix-assisted laser desorption/ionization (MALDI)-MS [4,5] capable of analysing intact globin chains. Pathological samples with a single mutation in the $\mathrm{Hb} \beta$-chain can thus be unambiguously classified into three groups: heterozygotes without $\mathrm{HbS}$ variants, heterozygotes with $\mathrm{HbS}$ variants and $\mathrm{HbS}$ homozygotes [5]. Furthermore, $\beta$-globin production defects ( $\beta$-thalassemia) can be detected [5]. The second tier is based on standard methods (such as HPLC, isoelectric focusing, and capillary electrophoresis (CE)) in order to distinguish between heterozygotes $\mathrm{Hb} \mathrm{F} / \mathrm{AS}$ (FAS) and compound heterozygotes (F/SC, F/SD, F/SE and F/S-OArab). In a single-tier procedure, only the most common variants (e.g., $\mathrm{HbS}, \mathrm{HbC}, \mathrm{D}^{\text {Punjab }}, \mathrm{OArab}$ and $\mathrm{HbE}$ ) are detected in a tryptic peptide analysis of haemoglobin using ESI-MS/MS [4,6-8] or the direct surface sampling of dried blood spots coupled to high-resolution MS [9-11].

In a multicentre pilot study, we evaluated a two-tier newborn SCD screening procedure in which the first tier is based on the fully automated MALDI-TOF MS classification of $\mathrm{Hb}$ profiles as FA, FS or FAS. To achieve this "plug-and-play" first tier, we maximised the performance levels of our previously described preanalytical and analytical procedures [5]. Likewise, we improved the software used for automatic data interpretation. Lastly, a laboratory information management system was set up to ensure data traceability. We investigated the usability of this MALDI-TOF MS newborn SCD screening platform by assessing its analytical throughput, user-friendliness, ease of implementation, potential interfering factors, MS spectral quality and percentage of correctly classified samples. We established quality criteria for standard and nonstandard MS profiles as a function of the newborn's phenotype, determined our algorithm's ability to correctly classify MS profiles and identified interfering factors and their causes. Lastly, we performed a preliminary analysis of the impact of $\beta$ chain variants other than $\mathrm{HbS}$ on the MALDI-TOF profile. Newborns having received one or more blood transfusions were excluded from the present analysis, and are being assessed in a separate, dedicated study. Our results demonstrated the potential value of a MALDI-TOF MS approach for high-throughput newborn SCD screening.

\section{Materials and Methods}

\subsection{Sample Collection}

Residual blood spots from standard Guthrie cards (used in our laboratory's routine screening activity) were investigated with MALDI-TOF MS. All samples were collected with the parents' consent for use in the French national newborn screening programme. No additional consent was required because the specimens were not used for purposes other than for which the blood sample was initially collected. The source of the samples has been described previously [5]. The study was registered with the French National Consultative Committee on Information Processing in Medical Research (Comité consultatif sur le traitement de l'Information en matière de recherche dans le domaine de la santé; reference: $14.81812 / 23 / 2014)$. After collection, the samples were anonymised and stored at $+4{ }^{\circ} \mathrm{C}$.

Sets of samples from our routine activity were selected each week, according to the following criteria: (i) samples presenting a $\beta$ chain variant in CE and HPLC assays; (ii) samples from premature newborns (gestational age at delivery: 22-32 weeks); (iii) samples corresponding to late and very late screening; and (iv) and randomly selected samples of daily screening activity that had an interpretable CE profile.

We analyzed 6701 samples with full datasets (i.e., with clinical data as follows: $\mathrm{N}^{\circ}$ of sample, sex, term, weight, name of maternity, transfusion, maternity location and date of screening, birth date plus validated results using CE in the first line and HPLC in the second line; Table 1). The percentage of FAS and FS phenotypes was higher for the premature newborns than for a standard population of newborns. The sample collection date was optimal (3-5 days after delivery) for the great majority of newborns. 
Table 1. Characteristics of the newborns and samples.

\begin{tabular}{|c|c|c|c|c|c|c|c|c|}
\hline \multirow{3}{*}{ Phenotype } & \multicolumn{8}{|c|}{ Characteristics of the Neonates and Samples } \\
\hline & \multirow{2}{*}{ Number } & \multicolumn{3}{|c|}{ Term } & \multicolumn{4}{|c|}{ Sample Collection Date } \\
\hline & & 23-32 weeks & 33-36 weeks & $>37$ weeks & 3-5 day & 6-10 day & 11-30 day & $>30$ day \\
\hline FS & 71 & 0 & 5 & 66 & 67 & 3 & 1 & 0 \\
\hline $\mathrm{F} / \mathrm{A}$ & 2834 & 203 & 809 & 1822 & 2651 & 90 & 77 & 16 \\
\hline $\mathrm{F} / \mathrm{AC}$ & 576 & 13 & 50 & 513 & 547 & 20 & 2 & 7 \\
\hline $\mathrm{F} / \mathrm{AE}$ & 141 & 2 & 9 & 130 & 138 & 2 & 0 & 1 \\
\hline F/AO-Arab & 21 & 0 & 2 & 19 & 19 & 2 & 0 & 0 \\
\hline $\mathrm{F} / \mathrm{AD}$ & 17 & 0 & 0 & 17 & 17 & 0 & 0 & 0 \\
\hline F/AKorle-Bu & 10 & 0 & 0 & 10 & 10 & 0 & 0 & 0 \\
\hline $\mathrm{F} / \mathrm{AX}$ & 86 & 4 & 3 & 79 & 81 & 3 & 0 & 2 \\
\hline $\mathrm{F} / \mathrm{C}$ & 7 & 0 & 1 & 6 & 6 & 0 & 0 & 1 \\
\hline $\mathrm{F} / \mathrm{E}$ & 3 & 0 & 0 & 3 & 2 & 1 & 0 & 0 \\
\hline F/O-Arab & 1 & 0 & 0 & 1 & 1 & 0 & 0 & 0 \\
\hline Corrected FA & 3696 & 222 & 874 & 2600 & 3472 & 118 & 79 & \\
\hline F/AS & 2894 & 57 & 247 & 2590 & 2763 & 89 & 23 & 19 \\
\hline $\mathrm{F} / \mathrm{SC}$ & 23 & 0 & 1 & 22 & 22 & 1 & 0 & 0 \\
\hline $\mathrm{F} / \mathrm{SE}$ & 1 & 0 & 0 & 1 & 1 & 0 & 0 & 0 \\
\hline F/SO-Arab & 1 & 0 & 1 & 0 & 1 & 0 & 0 & 0 \\
\hline Corrected FAS & 2919 & 57 & 249 & 2613 & 2787 & 90 & 23 & 19 \\
\hline S- $\beta^{+}$-thalassaemia & 15 & 0 & 1 & 14 & 15 & 0 & 0 & 0 \\
\hline \multicolumn{9}{|c|}{$\begin{array}{l}\text { The number of corrected FA phenotype corresponding to the sum of all samples (FA, FAC, FAE, FAO-Arab, FAD, } \\
\text { FAKorle-Bu, FAX, FC, FE, FO Arab where either } \beta^{\mathrm{A}} \text { gene was duplicated }\left[\beta^{\mathrm{A}} \beta^{\mathrm{A}}\right] \text { or associated with other } \beta \text { chain } \\
\text { variants }\left(\beta^{\mathrm{A}} \beta^{\mathrm{C}}, \beta^{\mathrm{A}} \beta^{\mathrm{E}}, \beta^{\mathrm{A}} \beta^{\mathrm{O}-A r a b}, \beta^{\mathrm{A}} \beta^{\mathrm{D}}, \beta^{\mathrm{A}} \beta^{\mathrm{C}}, \beta^{\mathrm{A}} \beta^{\mathrm{X}}\right) \text { or where duplicate } B \text { chain variants were found }\left(\beta^{\mathrm{C}} \beta^{\mathrm{C}} \text {, }\right. \\
\left.\beta^{\mathrm{E}} \beta^{\mathrm{E}}, \beta^{\mathrm{O} A r a b} \beta^{\mathrm{O}-A r a b}\right) \text { that are detected FA by MALDI. The number of corrected FAS phenotype corresponding } \\
\text { to the sum of all heterozygous (FAS) and composite heterozygous (FSC, FSE, FSO-Arab) samples where } \beta S \text { gene } \\
\text { was associated respectively to } \beta \mathrm{A} \text { gene or to other } \beta \text { chain variants }\left(\beta^{\mathrm{S}} \beta^{\mathrm{C}}, \beta^{\mathrm{S}} \beta^{\mathrm{E}}, \beta^{\mathrm{S}} \beta^{\mathrm{O}-\mathrm{Arab}}\right) \text { that are detected FAS } \\
\text { by MALDI. }\end{array}$} \\
\hline
\end{tabular}

\subsection{Definition of Corrected Phenotypes}

Subjects who were heterozygous or homozygous for $\mathrm{HbC}$, HbD-Punjab, $\mathrm{HbE}, \mathrm{HbO}-\mathrm{Arab}$, $\mathrm{HbKorle-Bu}$ and some unidentified variants $(\mathrm{HbX})$ according to conventional methods were detected as $\mathrm{HbA}$ by linear MALDI-TOF due to the technique's lack of mass resolution. Hence, these newborns were classified as having a corrected FA phenotype. Likewise, corrected FAS phenotypes included the composite heterozygous FSC, FSE and FS-OArab phenotypes.

\subsection{Sample Processing and Analysis}

In the present pilot study, all samples were measured with MALDI-MS at two analytical facilities (the University of Burgundy's CLIPP facility (Dijon, France) and Lille University Hospital's newborn screening laboratory (Lille, France)) after two chips (diameter: $3.5 \mathrm{~mm}$ ) had been isolated from each blood spot.

\subsection{Sample Preparation for MS Measurements}

At both facilities, samples were prepared for MS measurements using a research version of the NeoSickle ${ }^{\circledR}$ kit (Biomaneo, Dijon, France) for an EVO 200 automated system in Dijon and an EVO 100 automated system in Lille (Tecan, Lyon, France). The NeoSickle ${ }^{\circledR}$ kit was used according to the manufacturer's instructions, which included a first step of solubilization of proteins by a specific solution, and a second step of mixing with the matrix that was specially adapted for the MALDI-TOF MS analysis of blood proteins. The sample-matrix mixture was then deposited in quadruplicate on a 384-spot polished steel MALDI target (Bruker Daltonik GmbH, Bremen, Germany).

\subsection{Mass Spectrometry Measurements}

At both facilities, MS was performed with a MALDI-TOF system (an AutoFlex ${ }^{\mathrm{TM}}$ Speed with a $2000 \mathrm{~Hz}$ Smartbeam ${ }^{\mathrm{TM}}$ II laser (Bruker Daltonik GmbH) in Dijon, and an AutoFlex ${ }^{\mathrm{TM}}$ III with a $200 \mathrm{~Hz}$ Smartbeam ${ }^{\mathrm{TM}}$ laser (Bruker Daltonik $\mathrm{GmbH}$ ) in Lille. 


\subsection{Data Processing}

Mass spectrometry acquisitions were analyzed by the algorithm if (i) the whole spectrum and the region of interest were sufficiently intense, (ii) the baseline was not too noisy and (iii) at least three of the four profiles per sample could be interpreted automatically. After evaluation of the quality of each spectrum, the spectra were normalized, smoothed and underwent baseline subtraction. The four spectra obtained of the same sample were then averaged to obtain a mean profile, which was submitted to the algorithm.

\subsection{Analytical Data Flow}

An algorithm for automatic discrimination between normal samples and samples containing an $\mathrm{HbS}$ variant had been developed using spectra from an initial cohort of phenotyped samples by $\mathrm{CE}$ and HPLC. It has since been improved as further data are acquired. All of the analytical results were centralized via a secure data collector and submitted to the algorithm. The median newborns' profiles were automatically classified as FA, FAS or FS.

\subsection{Visual Assessment of MS Profiles}

In order to evaluate the algorithm's ability to classify a newborn's profile as FA, FAS or FS, all of the following profiles were visually inspected: (i) samples classified as FS or FAS by CE/HPLC, (ii) samples for which MALDI-MS and CE/HPLC gave conflicting results, (iii) samples with abnormal CE and/or HPLC profiles, (iv) samples from premature newborns, (v) samples with $\beta$ chain variants other than $\mathrm{HbS}$, (vi) samples with low-intensity MS signals at 15,850 $\mathrm{m} / \mathrm{z}$ and 15,880 $\mathrm{m} / \mathrm{z}$ [5], (vii) samples with a "low $\mathrm{HbA}$ " warning and (viii) samples for which the Dijon and Lille facilities gave conflicting results. Lastly, half the remaining samples (classified as FA by both HPLC and CE) were checked at random.

\subsection{The Data Collector}

Sickle Cell Anemia Collect \& Compare (SCACC) is a web application developed by Biomaneo to help biologists compare MS screening results with those of the reference screening methods (CE and HPLC). Within a single application, SCACC can store, group and present heterogeneous data in a user-friendly way, including clinical data on the sample donor, the experimental data from $\mathrm{CE}$ and HPLC analyses, the experimental data from the MS analysis and the validation files (i.e., the screening results sent to the paediatrician).

The SCACC application contains a table in which all of the information available for a given sample is shown on a single line. Along with a filter system for each variable, this layout makes it easy to create pools of interest (preterm samples, pathologic results, etc.) for the analysis of any misinterpreted results.

\section{Results}

\subsection{Optimization of Preanalytical and Analytical Procedures}

In France, newborn SCD screening is centralized at a few specialist analytical centres. This centralisation requires a very-high-throughput system capable of analyzing 600-1000 samples per day. The MALDI-TOF MS approach meets this requirement: for each run, it takes $1 \mathrm{~h}$ to deposit 288 samples in quadruplicate on three 384-spot MALDI targets.

Using an AutoFlex ${ }^{\mathrm{TM}}$ Speed system, it took 22 min to analyze a MALDI target containing 96 samples. Hence, the analytical throughput was estimated to be around 210 samples/hour; it took $5 \mathrm{~h}$ to analyze 1056 samples.

One of the essential criteria for automation was easy operation of the software that controlled the platform and displayed the results. A technician with little experience of MS could perform analyses on the MALDI-TOF MS system interfaced with the workstation. Another strong point is that all of the data 
from an analytical run were published and aggregated into a 96-well plate format. Abnormal profiles were differentiated from normal profiles by a colour code. Three different icons can be showed within a given coloured square: a cross indicating that the profile could not be interpreted, an exclamation mark indicating that the profile's initial automatic classification has been modified manually or a question mark indicating that the profile did not match the phenotype and must be checked by the operator. The spectrum's region of interest can be displayed in a mode in which $\beta, \beta S$ and $\gamma$ chains are seen or in a zoom mode in which only the $\beta$ and $\beta S$ chains are seen (Figure 1). The abnormal MS profiles were assessed visually by the operator. When applicable, two decision-support warnings could be displayed by the algorithm: "low $\mathrm{HbA}$ concentration" or "newborn having received a blood transfusion". The data for a given newborn (ID number, demographic data, zoom mode or not, classification changes and non-hidden alerts) can be displayed by clicking on a well.

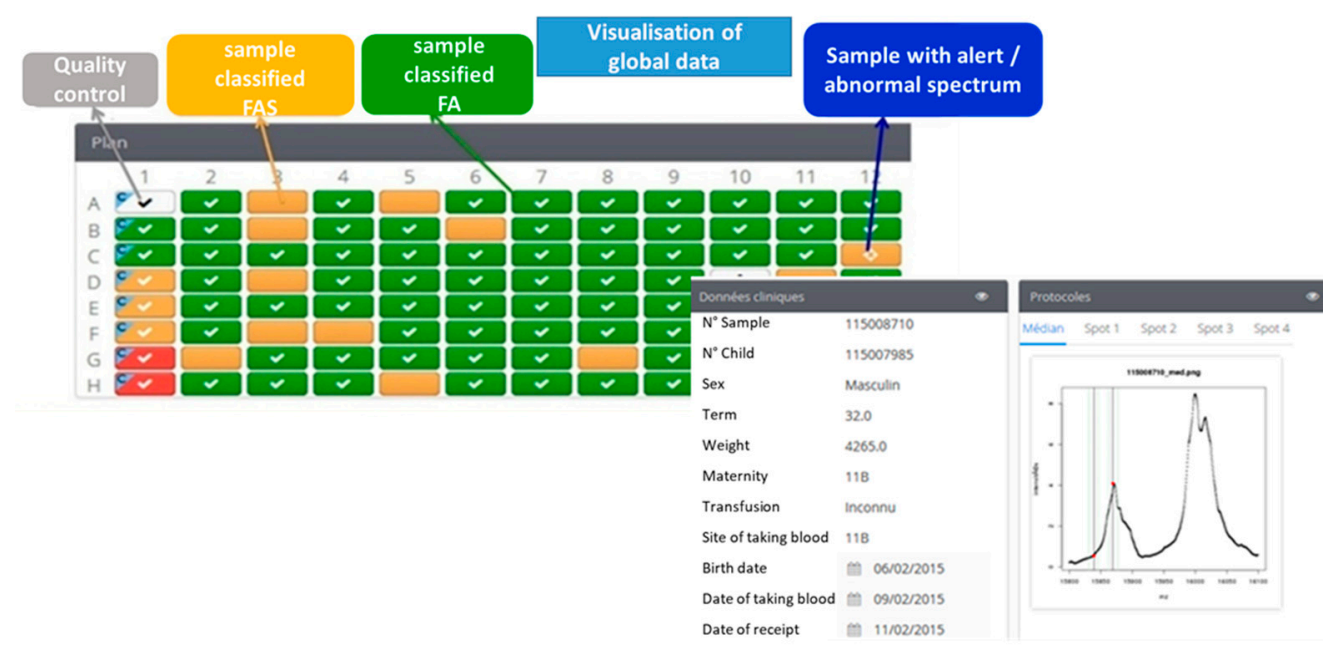

Figure 1. A screen display from the Sickle Cell Anemia Collect \& Compare (SCACC) web application. Each box corresponds to a position of a sample on the 96-well plate. The green box corresponds to one sample classified FA, the orange box corresponds to one sample classified FAS and the red boxes are samples detected as FS. The software presents on the same page the clinical data $\left(\mathrm{N}^{\circ}\right.$ of sample, sex, term, weight, name of maternity, transfusion, location and date of taking blood, birth date) and the spectra of one sample.

\subsection{Spectral Quality of MALDI-TOF Data}

Taken as a whole, our results show NeoSickle ${ }^{\circledR \prime}$ s ability to correctly classify $97 \%$ of the samples tested in Lille and $98.8 \%$ of the samples tested in Dijon. The sensitivity of the NeoSickle ${ }^{\circledR}$ approaches to detect the FA samples is $99.2 \%$ and $97.2 \%$ with a specificity of $99 \%$ and $99.3 \%$ on the Lille and the Dijon MS facility, respectively. The detection of an HbS chain in samples is obtained on the Lille and the Dijon MS facility with a sensitivity of $98.4 \%$ and $97 \%$ and with a specificity of $99.8 \%$ and $99 \%$, respectively.

The positive predictive value of FS profiles detection was $96.6 \%$ and $97.7 \%$ with the results obtained by the Lille and the Dijon MS facility, respectively. The negative predictive value of FS profiles detection obtained at Lille and the Dijon MS facility was 100\% and $99.9 \%$, respectively.

It is important to note that these results were obtained with biased blood samples that were enriched in FS and FAS samples and difficult samples (premature newborns, very late screening).

\subsubsection{Criteria Used to Define a Standard MALDI-TOF Profile}

In $85.8 \%$ of the analyses in Dijon and $92.9 \%$ of the analyses in Lille, the median FA, FAS, FS and S- $\beta^{+}$MS profiles (Figure $2 \mathrm{a}, \mathrm{c}, \mathrm{e}, \mathrm{g}$, respectively) were considered to be standard and were automatically interpreted by the NeoSickle ${ }^{\circledR}$ software. Limits not to be exceeded were defined: an FA profile with a low-noise ascending or convex baseline was considered to be normal (Figure 2b); an FAS profile was considered to be standard as long as a plateau separated the $\beta^{\mathrm{S}}$ and $\beta^{\mathrm{A}}$ chain peaks (Figure 2d); 
and, lastly, an FS profile with a low-noise ascending or convex baseline to the right of the $\beta^{S}$ chain peak was considered to be interpretable (Figure $2 \mathrm{f}$ ). The profile of a newborn with S- $\beta^{+}$-thalassemia was characterized by a much more intense $\beta^{S}$ peak, relative to the $\beta^{A}$ peak (Figure $2 \mathrm{~g}, \mathrm{~h}$ ).
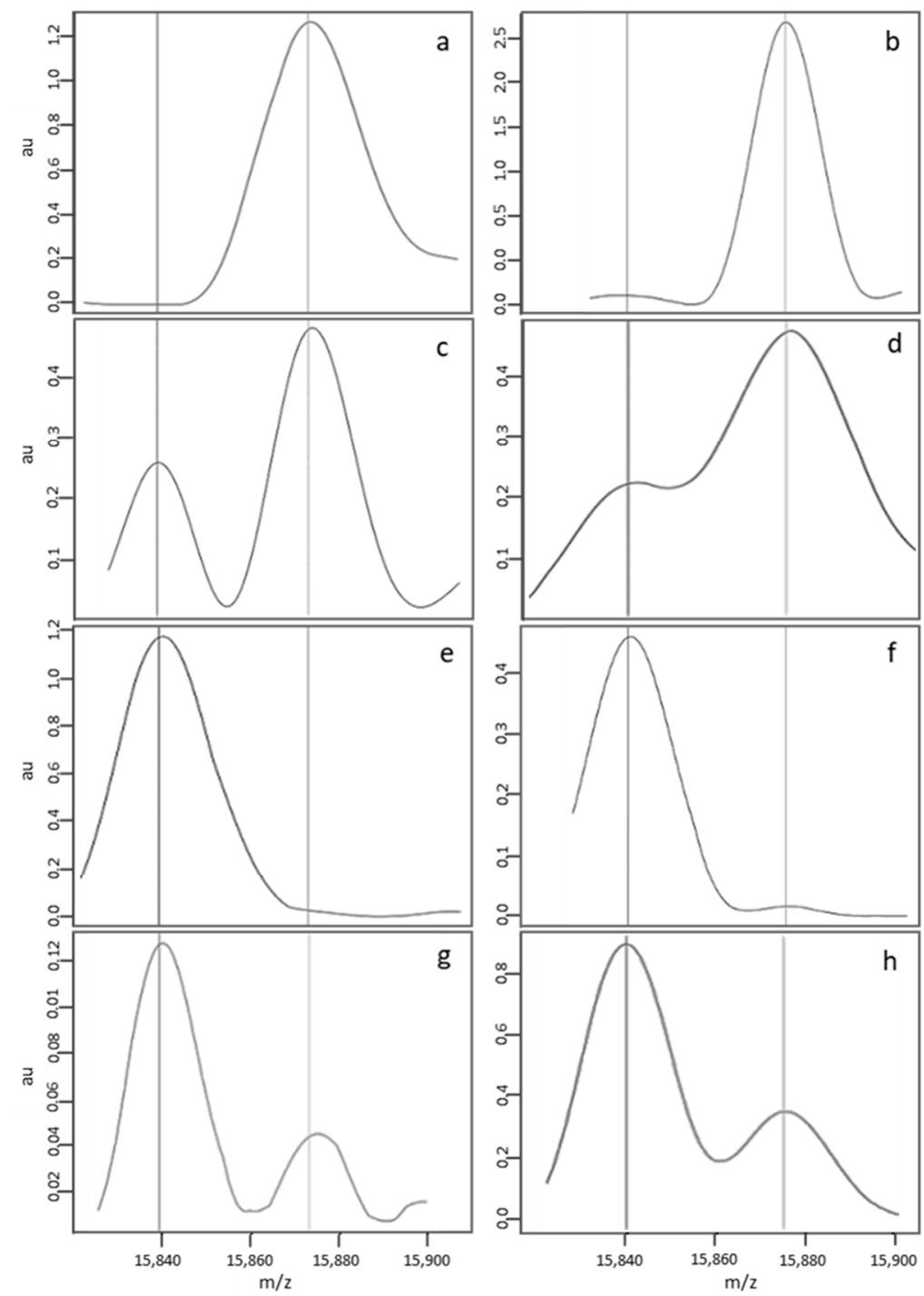

Figure 2. Examples of standard MS profiles correctly classified by the NeoSickle ${ }^{\circledR}$ software, from newborns with the following phenotypes: FA $(\mathbf{a}, \mathbf{b})$, FAS $(\mathbf{c}, \mathbf{d})$, FS $(\mathbf{e}, \mathbf{f})$ and S- $\beta+(\mathbf{g}, \mathbf{h})$.

3.2.2. Classification by the Algorithm of "Standard" Profiles, as a Function of the Newborn's Corrected Phenotype

There were no classification errors among the corrected FA and FS samples (Table 2). Concerning the corrected FAS samples, only 2 of the 2753 standard profiles from Lille were misclassified (as FS) by MS, and 17 of the 2507 standard profiles from Dijon were misclassified (16 cases classified as FA by MS, and 1 case classified as FS by MS). Although the spectra of the FAS samples misclassified as FA showed a shoulder with a plateau at $15,850 \mathrm{~m} / z \pm 10 \mathrm{~m} / \mathrm{z}$, the signal intensity was too low. This characteristic should be introduced in the criteria for a nonstandard MS profile.

The FAS samples misclassified as FS by MALDI-MS came from composite heterozygous newborns classified as FS-O Arab and FSE by CE and HPLC. Regular peaks at 18,850 and $1880 \mathrm{~m} / \mathrm{z}$ were observed; however, the error was induced by the low intensity of the peak at $15,880 \mathrm{~m} / \mathrm{z}$. A profile with asymmetry for the $\beta^{\mathrm{A}}$ and $\beta^{\mathrm{S}}$ chains (a more intense $\beta^{\mathrm{S}}$ peak) prompted its classification as FS by the e-NeoSickle software. 
Table 2. The number of standard MS profiles correctly classified (+) or misclassified ( - ), according to the newborn's corrected phenotype.

\begin{tabular}{|c|c|c|c|c|c|c|c|c|c|c|c|c|}
\hline & \multicolumn{6}{|c|}{ Lille MS Facility } & \multicolumn{6}{|c|}{ Dijon MS Facility } \\
\hline Number of Standard Profiles & \multicolumn{6}{|c|}{6181} & \multicolumn{6}{|c|}{5698} \\
\hline Corrected Phenotype & \multicolumn{2}{|c|}{ FA } & \multicolumn{2}{|c|}{ FAS } & \multicolumn{2}{|c|}{ FS } & \multicolumn{2}{|c|}{ FA } & \multicolumn{2}{|c|}{ FAS } & \multicolumn{2}{|c|}{ FS } \\
\hline Number of samples & \multicolumn{2}{|c|}{3386} & \multicolumn{2}{|c|}{2753} & \multicolumn{2}{|c|}{37} & \multicolumn{2}{|c|}{3161} & \multicolumn{2}{|c|}{2507} & \multicolumn{2}{|c|}{35} \\
\hline \multirow{2}{*}{ classification by the algorithm } & + & - & + & - & + & - & + & - & + & - & + & - \\
\hline & 3386 & 0 & 2751 & 2 & 37 & 0 & 3161 & 0 & 2490 & 17 & 35 & 0 \\
\hline
\end{tabular}

The 16 samples misclassified in Dijon gave good results in Lille, indicating that the errors were probably due to a temporary lack of spectrum quality and not a sample or detection problem. The quality of the Dijon data was not as high as in Lille because the number of nonstandard spectra was higher; this finding agrees with the higher misclassification rate observed in Dijon.

\subsubsection{Description of a Nonstandard MALDI-MS Profile}

A nonstandard median MS profile was detected in $14.7 \%$ of the analyses in Dijon and $7.6 \%$ of the analyses in Lille. The MS profiles were considered to be nonstandard (Figure 3) for one of three reasons. Firstly (cause 1, see Table 3), some profiles had a nonregular baseline or a slightly distorted peak; this applied to (i) FA MS profiles with a variably broad/sharp/distorted peak but very low intensity at $15,837 \mathrm{~m} / z \pm 5 \mathrm{Da}$, relative to the $\beta^{\mathrm{A}}$ peak (Figure $3 \mathrm{a}, \mathrm{b}$ ); an FAS MS profile with minor deformation of the $\beta^{S}$ peak (Figure 3c); and FS and S- $\beta^{+}$-thalassemia MS profiles with an irregular baseline (data not shown) and a deformation of the $\beta^{\mathrm{A}}$ peak (Figure 3d). Secondly (cause 2), some profiles had a low, broad peak at $15,837 \mathrm{~m} / \mathrm{z}$; this applied to (i) FA MS profiles with a variably shifted peak or (ii) FAS MS profiles characterized by a broad, well-centred peak at 15,837 $\pm 5 \mathrm{~m} / \mathrm{z}$ but that was much less intense than the $\beta^{A}$ chain peak at $15,867 \mathrm{~m} / z$ (data not shown). Thirdly (cause 3), some FA MS profiles had a broader $\beta^{\mathrm{A}}$ peak that variably overlapped with the region of interest at 15,837 $m / z$ and led to misclassification as "FAS" (Figure 3e), whereas other FAS MS profiles showed a low resolution and thus poor separation of the $\beta^{\mathrm{A}}$ and $\beta^{\mathrm{S}}$ peaks, giving a single large peak as a shoulder and no plateau between the peaks (Figure 3f).
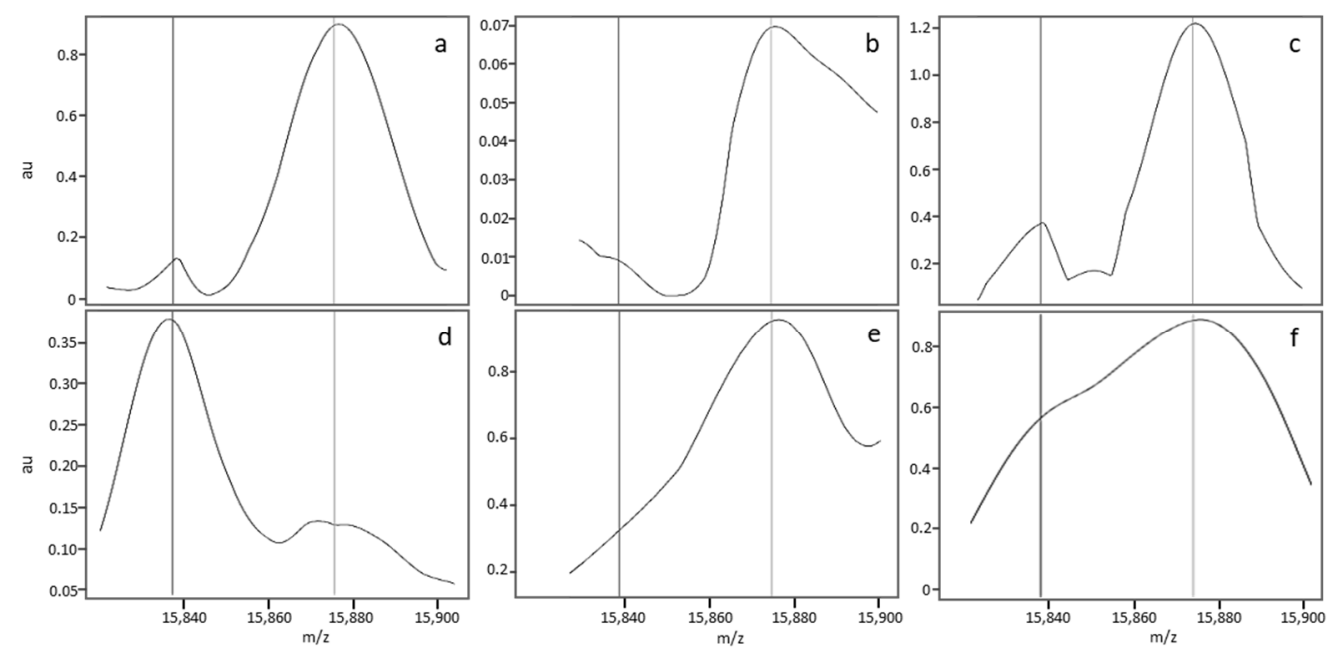

Figure 3. Zoom of MS profiles on the zone of interest for nonstandard FA $(\mathbf{a}, \mathbf{b}, \mathbf{e})$, FAS $(\mathbf{c}, \mathbf{f})$ and FS (d) samples. 
3.2.4. Classification by the Algorithm of Profiles Considered to be Nonstandard in a Visual Assessment, as a Function of the Newborn's Corrected Phenotype

The data in Table 3 show that the great majority of nonstandard profiles $(84.5 \%$ in Lille and $81.2 \%$ in Dijon) were nevertheless correctly classified by the e-NeoSickle ${ }^{\circledR}$ algorithm. Furthermore, $7.5 \%$ of the nonstandard profiles in Lille and $4.6 \%$ of the nonstandard profiles Dijon were misclassified, and $8 \%$ of the nonstandard profiles in Lille and $14.2 \%$ of the nonstandard profiles in Dijon were uninterpretable.

It is important to note that the nonstandard profiles accounted for less than $14 \%$ of the analyses in Dijon and $7 \%$ of the analyses in Lille. Overall, only $2 \%$ of the profiles in Dijon and $0.6 \%$ of the profiles in Lille were uninterpretable, and $0.9 \%$ of the profiles in Dijon and $0.6 \%$ of the profiles in Lille were misclassified.

Table 3. Classification by the algorithm of profiles considered to be nonstandard in a visual assessment. Some spectra could not be interpreted by the algorithm (IN). The percentage is based on the number of profiles considered to be nonstandard (780 profiles at Lille and 985 at Dijon).

\begin{tabular}{|c|c|c|c|c|c|}
\hline & & & \multicolumn{3}{|c|}{ Corrected Phenotypes } \\
\hline & & & FA & FAS & FS \\
\hline \multirow{8}{*}{$\begin{array}{l}\text { MALDI-TOF MS classification of } \\
\text { non-standard profiles }\end{array}$} & \multirow{4}{*}{ Lille } & FA & $282(54.7 \%)$ & $30(5.9 \%)$ & $0(0 \%)$ \\
\hline & & FAS & $7(1.4 \%)$ & $118(23.2 \%)$ & $0(0 \%)$ \\
\hline & & FS & $0(0 \%)$ & $1(0.2 \%)$ & $34(6.7 \%)$ \\
\hline & & IN & $21(4.5 \%)$ & $17(3.4 \%)$ & $0(0 \%)$ \\
\hline & \multirow{4}{*}{ Dijon } & FA & $446(45 \%)$ & $22(2.2 \%)$ & $0(0 \%)$ \\
\hline & & FAS & $21(2.2 \%)$ & $323(32.7 \%)$ & $1(0.2 \%)$ \\
\hline & & FS & $0(0 \%)$ & $1(0.2 \%)$ & $36(3.7 \%)$ \\
\hline & & IN & $68(7.2 \%)$ & $67(6.8 \%)$ & $0(0 \%)$ \\
\hline
\end{tabular}

The frequencies of "nonregular baseline" (cause 1) and "low/broad peak" (cause 2) features were similar at the two MS facilities. Our analysis showed that most of the nonstandard spectra in Dijon (54\%) were due to a lack of resolution (cause 3), whereas this was the case for only $13 \%$ in Lille. Proportionally, the corrected phenotype FS $(n=71)$ was more likely to be labelled as "low resolution", with respectively 31 and 36 abnormal spectra in Lille and Dijon. However, this low resolution had very little impact on the automatic classification, since less than $1 \%$ of all spectra labelled as "low resolution" were misclassified.

Sixteen percent of the spectra with a low, broad peak at $15,837 \mathrm{~m} / \mathrm{z}$ were misclassified by the algorithm (Table 4). Of the 21 spectra of this type misclassified in Lille, 20 (95\%) came from newborns with a corrected FAS phenotype. Likewise, of the 20 spectra of this type misclassified in Dijon (Table 4), 18 (90\%) came from newborns with a corrected FAS phenotype. These spectra were very difficult to classify because the ratio between the $\beta^{S}$ and $\beta^{A}$ peaks was abnormal. A very low $\beta^{S}$ peak (relative to the $\beta^{A}$ peak) can be interpreted as a sample with high background noise or as a sample from an $S$ homozygote or an AS heterozygote having received a blood transfusion (which induces a strong imbalance). Given that these spectra have low peak intensities, we recommend repeating the analysis or depositing a new sample. 
Table 4. (A) Numbers and proportions of nonstandard spectra by corrected phenotype and by cause. (B) Numbers and proportions of correctly classified and misclassified spectra by cause. Cause 1 resulted in spectra with an irregular baseline, cause 2 resulted in spectra with a low, broad peak and cause 3 resulted in spectra with a low resolution. The percentage is based on the number of profiles considered to be nonstandard (780 profiles at Lille and 985 at Dijon).

\begin{tabular}{|c|c|c|c|c|c|c|c|c|}
\hline & \multirow{2}{*}{ A } & \multicolumn{3}{|c|}{ Corrected Phenoztype } & & \multirow{2}{*}{ B } & \multirow{2}{*}{ Correctly Classified } & \multirow{2}{*}{ Misclassified } \\
\hline & & FA & FAS & FS & & & & \\
\hline \multirow{3}{*}{ Lille } & Cause 1 & $179(37 \%)$ & $90(19 \%)$ & $3(0 \%)$ & \multirow{3}{*}{ Lille } & Cause 1 & $252(94 \%)$ & $17(6 \%)$ \\
\hline & Cause 2 & $98(21 \%)$ & $28(5 \%)$ & $0(0 \%)$ & & Cause 2 & $105(84 \%)$ & $21(16 \%)$ \\
\hline & Cause 3 & $12(3 \%)$ & $31(7 \%)$ & $31(7 \%)$ & & Cause 3 & $77(100 \%)$ & $0(0 \%)$ \\
\hline \multirow{3}{*}{ Dijon } & Cause 1 & $193(23 \%)$ & $69(8 \%)$ & $0(0 \%)$ & \multirow{3}{*}{ Dijon } & Cause 1 & $248(95 \%)$ & $14(5 \%)$ \\
\hline & Cause 2 & $103(12 \%)$ & $24(3 \%)$ & $0(0 \%)$ & & Cause 2 & $107(84 \%)$ & $20(16 \%)$ \\
\hline & Cause 3 & $171(20 \%)$ & $253(30 \%)$ & $36(4 \%)$ & & Cause 3 & $449(98 \%)$ & $11(2 \%)$ \\
\hline
\end{tabular}

\subsubsection{Causes of Abnormal Spectral Features in Nonstandard MS Profiles}

A spectral analysis showed that nonstandard profiles mainly resulted from the compilation of differing spectra within a quadruplicate. Figure 4 shows the variability in the raw data and its impact on data processing. The spectra in Figure $4 a, b, e, f$ correspond to the raw data generated for the first (Figure $4 a, b$ ) and third (Figure 4e,f) of the quadruplicate sample depositions. The two depositions varied markedly in terms of the raw spectral intensity (less than 40 ua for deposition 1, and 200 ua for deposition 3). The data processing steps (including peak alignment and baseline normalisation and subtraction) normalise the spectrum intensity but also remove differences (see Figure $4 \mathrm{c}, \mathrm{g}$, corresponding to the normalized spectra, and a blow-up view of the zone of interest Figure $4 \mathrm{~d}, \mathrm{~h}$ ). The compilation of normalized data introduces a bias, and might lead to misinterpretation of the results by the software. Figure 4i shows a median spectrum with an irregular baseline; this "pseudo $\beta^{S}$ peak" can be confused with a true $\beta^{S}$ peak. However, the relative intensity of the pseudo $\beta^{S}$ peak differed from that of a true $\beta^{A}$ peak.

The analysis of each individual spectrum enabled us to affirm that at least one good-quality, high-resolution spectrum was obtained for each biological sample. The sample deposition method for MALDI-MS is known not to be highly reproducible, and thus makes it difficult to obtain very homogeneous results. Even though our study highlighted this difficulty, our analysis also showed that a high-quality result can be obtained systematically. 

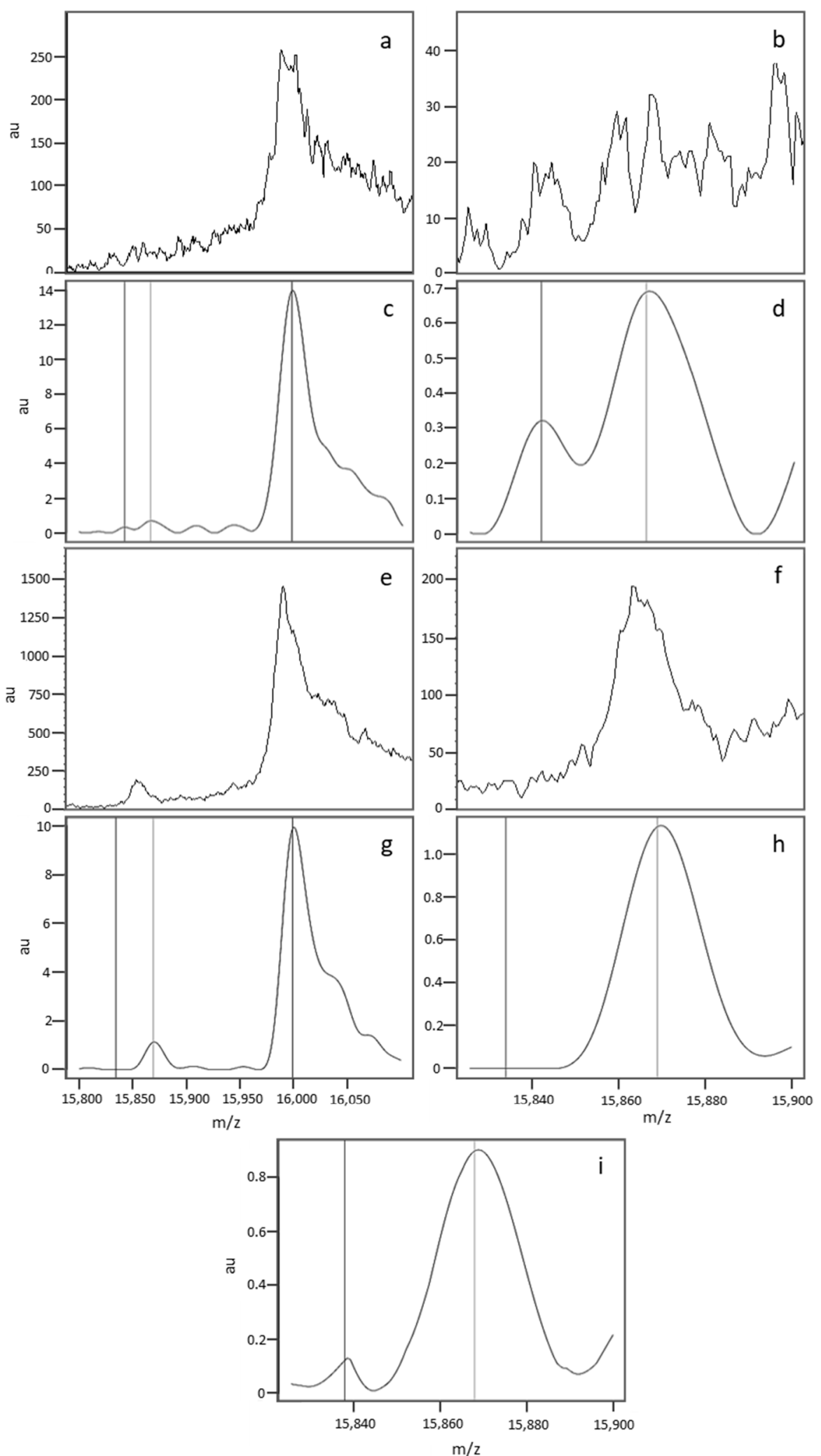

Figure 4. The spectra in $(\mathbf{a}, \mathbf{b}, \mathbf{e}, \mathbf{f})$ correspond to the raw data generated for the first $(\mathbf{a}, \mathbf{b})$ and third $(\mathbf{e}, \mathbf{f})$ of the quadruplicate depositions from the same sample. (a,c,e,g) show the whole spectrum $(15,800-16,100 \mathrm{Da})$, and $(\mathbf{b}-\mathbf{f}, \mathbf{h}, \mathbf{i})$ show the region of interest $(15,820-15,900 \mathrm{Da})$. The spectra in (c-h correspond to the processed spectra after alignment, baseline subtraction, and normalisation. The median spectrum (i) was obtained by compiling the individual normalized spectra. 


\section{Occasional Misclassifications}

The analysis of samples that were classified differently by the two analytical facilities confirmed that MALDI-MS can provide an interpretable result for each sample. In fact, the data in Table 5 showed that the 68 samples with a corrected FA phenotype that could not be interpreted in Dijon were correctly classified in Lille (67 standard spectra and 1 low-resolution spectrum). Likewise, the 21 samples with a corrected FA phenotype and that could not be interpreted in Lille were correctly classified at Dijon. Identical results were obtained with the corrected FAS samples.

Table 5. Paired classification of the samples by the two analytical facilities. Relationship with the spectral quality. This table summarizes the number of profiles correctly classified, misclassified and uninterpretable for each category of profile quality and for the two analytical laboratories.

\begin{tabular}{|c|c|c|c|c|c|c|c|c|c|c|c|}
\hline \multicolumn{12}{|c|}{ Profile Abnormalities at $15,850 \pm 10 \mathrm{~m} / \mathrm{z}$} \\
\hline \multicolumn{12}{|c|}{ Neonates with a Corrected FA Phenotype } \\
\hline \multicolumn{2}{|c|}{$\begin{array}{l}\text { Automatic Classification of Matched } \\
\text { Profiles }\end{array}$} & \multicolumn{2}{|c|}{ Irregular Base Line } & \multicolumn{2}{|c|}{ Low, Broad Peak } & \multicolumn{2}{|c|}{ Low Resolution } & \multicolumn{2}{|c|}{ Regular Base Line } & \multicolumn{2}{|c|}{ Uninterpretable } \\
\hline Lille & Dijon & Lille & Dijon & Lille & Dijon & Lille & Dijon & Lille & Dijon & Lille & Dijon \\
\hline correctly classified & uninterpretable & 1 & 0 & 0 & 0 & 0 & 0 & 67 & 0 & 0 & 68 \\
\hline correctly classified & Misclassified & 4 & 10 & 7 & 0 & 1 & 5 & 17 & 0 & 0 & 0 \\
\hline uninterpretable & correctly classified & 0 & 0 & 0 & 0 & 0 & 0 & 0 & 21 & 0 & 0 \\
\hline Misclassified & correctly classified & 4 & 0 & 3 & 0 & 0 & 0 & 0 & 7 & 0 & 0 \\
\hline \multicolumn{12}{|c|}{ Neonates with a Corrected FAS Phenotype } \\
\hline \multicolumn{2}{|c|}{$\begin{array}{l}\text { Automatic Classification of Matched } \\
\text { Profiles }\end{array}$} & \multicolumn{2}{|c|}{ Irregular Base Line } & \multicolumn{2}{|c|}{ Low, Broad Peak } & \multicolumn{2}{|c|}{ Low Resolution } & \multicolumn{2}{|c|}{ Regular Base Line } & \multicolumn{2}{|c|}{ Uninterpretable } \\
\hline Lille & Dijon & Lille & Dijon & Lille & Dijon & Lille & Dijon & Lille & Dijon & Lille & Dijon \\
\hline correctly classified & uninterpretable & 0 & 0 & 0 & 0 & 0 & 0 & 68 & 0 & 0 & 68 \\
\hline correctly classified & Misclassified & 0 & 4 & 0 & 14 & 1 & 5 & 23 & 0 & 0 & 0 \\
\hline uninterpretable & correctly classified & 0 & 0 & 0 & 0 & 0 & 1 & 0 & 16 & 17 & 0 \\
\hline Misclassified & correctly classified & 12 & 0 & 12 & 0 & 0 & 4 & 6 & 26 & 0 & 0 \\
\hline
\end{tabular}

When a profile was misclassified or uninterpretable at one MS facility, the corresponding profile at the other MS facility was correctly classified and was less likely to be a nonstandard profile. Only one sample with a nonstandard profile was misclassified at both MS facilities (data not shown). A good classification with a standard spectrum was obtained for each sample on at least one of the MS facilities, which indicates that a low $\mathrm{HbA}$ concentration was not the main cause of misclassifications or uninterpretable results. Misclassifications or uninterpretable results were mainly due to the absence of a high-quality spectrum induced by technical incidents (tip blockage and non-deposition of the sample). Consequently, repeating the analysis of a sample with a nonstandard profile was the best way of obtaining a standard profile and a correct classification. Different data, such as peak intensities or the ratio between different peaks of $\beta^{\mathrm{A}}$ and $\beta^{S}$ chains, allow for us to detect the nonstandard profile and to add an alert to the results.

\section{Discussion}

Newborn disease screening programmes are front-line public health measures, and as such must be based on robust analytical methods and data-processing software. Cost effectiveness is a further requirement, prompting the implementation of high-throughput screening units that reduce unit costs. Lastly, the maximal use of automation enables the analytical results to be validated with as little intervention as possible by the medical team. Our MALDI-MS platform and the associated data-processing and interpretation software were designed to address these challenges.

In newborn SCD screening with a MALDI MS system, the user-friendliness of the software interface and a high-throughput analysis coupled to automatic sample classification and traceability are directly related to practicability. As mentioned above, newborn SCD screening in France is centralised at a few specialist analytical centres. This centralisation requires a throughput of up to 600-1000 samples per day. The MALDI-TOF MS approach meets this requirement.

The next performance criterion of note concerns the method's robustness: in other words, its ability to generate good-quality standard MS profiles that enable correct classification in agreement with the 
sample's validated phenotype, whatever the newborn's clinical status. In order to test our MALDI-MS under extreme conditions, we increased the proportion of difficult samples in the presently analyzed cohort; this corresponds to pathological specimens and specimens collected and/or stored under non-optimal conditions (samples from premature newborns, samples collected long after delivery, samples collected from France's overseas regions and dependencies that may have been stored for a long time in a tropical atmosphere before delivery to the screening laboratory in continental France, etc.). In the present study, only samples from newborns having received one or more blood transfusions were discarded. The impact of blood transfusion will be described in a specific study of the effect of high $\mathrm{HbA}$ levels on the method's sensitivity, specificity and resolution as a function of the $\beta^{\mathrm{s}}$ chain concentration. Moreover, we evaluated the MALDI-TOF method's robustness by visually checking the spectral quality as well as by considering its ability to correctly classify the newborns as FA, FAS or FS. Benchmark MALDI-TOF MS spectra were thus established on the basis of the classification results and the visual quality of the spectra. The reference spectra correspond to all of the spectra defined as standard for which the information obtained is considered to be sufficient and of good quality. Indeed, visual spectral validation has proved to be most effective for revealing shot-to-shot variations.

Using this strategy, we determined the percentages of newborns with a standard profile and who were correctly classified (relative to their validated phenotype). The highest level of efficiency was obtained in the set of newborns with a corrected FA phenotype; around $85 \%$ of these analyses could be validated directly. A similar percentage was obtained for newborns with a corrected FAS phenotype. Moreover, nonstandard MS spectra were not systematically misclassified, and most were correctly classified. We consider these results to be very promising because it should be possible to further improve the procedures for sample deposition, raw data analysis and selection of the mass spectra to which the classification algorithm is applied.

None of the samples from FS newborns were misclassified, and a sample with an FAS-corrected phenotype was misclassified as an FA sample. This type of misclassification can lead to false negatives for newborns with an FSC phenotype. This major error required us to set up a strategy for correcting the automatic classification. The development and characteristics of tracking alerts for the nonstandard MS profiles will be described elsewhere. This approach should enable an occasional lack of reproducibility, sensitivity or resolution in $\mathrm{Hb}$ profiling to be detected.

There were two main reasons for the incorrect automatic classification of newborn phenotypes. Firstly, a low signal intensity at 15,850 and/or $15,880 \mathrm{~m} / \mathrm{z}$ (i.e., similar to background noise) was intensified by the data processing (normalisation and alignment). Secondly, poor resolution of the $\beta$ and $/$ or $\beta^{S}$ chains generated signal overlap. The quadruplicate sample analysis improved the results, but was not always able to counterbalance shot-to-shot variability.

In a previous article [5], very poor spectral quality prevented the analysis of 20 of 844 samples (2.5\%), even after several repeat analyses. The relatively high frequency of this incident was probably due to the inclusion of a high proportion of samples from premature newborns and/or $\mathrm{HbS}$ carriers. We have now resolved this problem. In most cases, a single repeat analysis was enough to obtain a standard profile. Some problems arose from time to time but were mainly related to robotic preparation of the blood spot samples. Further optimization of sample processing and the MS acquisition protocol should further improve the standardization of the MS profiles and reduce the frequency of repeat analysis.

Taken as a whole, our results for pooled standard and nonstandard spectra demonstrated NeoSickle ${ }^{\circledR \prime} \mathrm{s}$ ability to classify correctly $97 \%$ of the samples tested in Lille and $98.8 \%$ of the samples tested in Dijon. Furthermore, only $2 \%$ of the nonstandard spectra in Dijon were uninterpretable, and less than $0.9 \%$ were misclassified. In Lille, $0.6 \%$ of the nonstandard spectra were uninterpretable and $0.6 \%$ were misclassified. It is important to note that these percentages were obtained with "difficult" blood samples (premature newborns, very late screening).

Our pilot study showed that the NeoSickle ${ }^{\circledR}$ approach can differentiate between heterozygous FSE, FSO-Arab and S- $\beta+$ samples on one hand and heterozygous FAS and homozygous FS samples on the other. Indeed, the signal intensity of the $\beta^{\mathrm{E}}, \beta^{\mathrm{O}-\mathrm{Arab}}$ variants was much weaker than that of the $\beta$ chain. 
In addition to classifying samples as FA, FAS or FS, studies of a larger cohort of patients might make it possible to clearly differentiate a fourth class (corresponding to heterozygous SX samples in which the $\beta^{X}$ chain is E, O-Arab, a).

In conclusion, our new MALDI-TOF MS approach already meets today's requirements [12] for large-scale, cost-effective newborn SCD screening, and is well-positioned to address future requirements for even greater throughputs and total automation to detect the HbS variants.

Author Contributions: D.R. and M.E.O.: MALDI-TOF methodology; P.N.: software development; G.R. and O.G.: validation; P.D. and J.-M.P.: conceptualization, data curation, acquisition of funding and drafting of the manuscript.

Funding: The present research was funded by Lille University Medical Center and the Conseil Régional de Bourgogne.

Conflicts of Interest: Patrick Ducoroy was an employee of the University of Burgundy at the time of the research described here. He then founded the company Biomaneo, and currently serves as its CEO.

\section{References}

1. Rai, D.K.; Griffiths, W.J.; Landin, B.; Wild, B.J;; Alvelius, G.; Green, B.N. Accurate mass measurement by electrospray ionization quadrupole mass spectrometry: Detection of variants differing by $<6 \mathrm{Da}$ from normal in human hemoglobin heterozygotes. Anal. Chem. 2003, 75, 1978-1982. [CrossRef] [PubMed]

2. Wild, B.J.; Green, B.N.; Cooper, E.K.; Lalloz, M.R.; Erten, S.; Stephens, A.D.; Layton, D.M. Rapid identification of hemoglobin variants by electrospray ionization mass spectrometry. Blood Cells Mol. Dis. 2001, 27, 691-704. [CrossRef] [PubMed]

3. Wild, B.J.; Green, B.N.; Stephens, A.D. The potential of electrospray ionization mass spectrometry for the diagnosis of hemoglobin variants found in newborn screening. Blood Cells Mol. Dis. 2004, 33, 308-317. [CrossRef] [PubMed]

4. Kiernan, U.A.; Black, J.A.; Williams, P.; Nelson, R.W. High-throughput analysis of hemoglobin from neonates using matrix-assisted laser desorption/ionization time-of-flight mass spectrometry. Clin. Chem. 2002, 48, 947-949. [PubMed]

5. Hachani, J.; Duban-Deweer, S.; Pottiez, G.; Renom, G.; Flahaut, C.; Périni, J.M. MALDI-TOF MS profiling as the first-tier screen for sickle cell disease in neonates: Matching throughput to objectives. Proteomics Clin. Appl. 2011, 5, 405-414. [CrossRef] [PubMed]

6. Daniel, Y.A.; Turner, C.; Haynes, R.M.; Hunt, B.J.; Dalton, R.N. Rapid and specific detection of clinically significant haemoglobinopathies using electrospray mass spectrometry-mass spectrometry. Br. J. Haematol. 2005, 130, 635-643. [CrossRef] [PubMed]

7. Boemer, F.; Ketelslegers, O.; Minon, J.M.; Bours, V.; Schoos, R. Newborn screening for sickle cell disease using tandem mass spectrometry. Clin. Chem. 2008, 54, 2036-2041. [CrossRef] [PubMed]

8. Moat, S.J.; Rees, D.; King, L.; Ifederu, A.; Harvey, K.; Hall, K.; Lloyd, G.; Morrell, C.; Hillier, S. Newborn blood spot screening for sickle cell disease by using tandem mass spectrometry: Implementation of a protocol to identify only the disease states of sickle cell disease. Clin. Chem. 2014, 60, 373-380. [CrossRef] [PubMed]

9. Edwards, R.L.; Griffiths, P.; Bunch, J.; Cooper, H.J. Compound heterozygotes and beta-thalassemia: Top-down mass spectrometry for detection of hemoglobinopathies. Proteomics 2014, 14, 1232-1238. [CrossRef] [PubMed]

10. Edwards, R.L.; Griffiths, P.; Bunch, J.; Cooper, H.J. Top-down proteomics and direct surface sampling of neonatal dried blood spots: Diagnosis of unknown hemoglobin variants. J. Am. Soc. Mass Spectrom. 2012, 23, 1921-1930. [CrossRef] [PubMed]

11. Edwards, R.L.; Creese, A.J.; Baumert, M.; Griffiths, P.; Bunch, J.; Cooper, H.J. Hemoglobin variant analysis via direct surface sampling of dried blood spots coupled with high-resolution mass spectrometry. Anal. Chem. 2011, 15, 2265-2270. [CrossRef] [PubMed]

12. Lobitz, S.; Telfer, P.; Cela, E.; Allaf, B.; Angastiniotis, M.; Backman Johansson, C.; Badens, C.; Bento, C.; Bouva, M.J.; Canatan, D.; et al. Newborn screening for sickle cell disease in Europe: Recommendations from a Pan-European consensus conference. Br. J. Haematol. 2018. [CrossRef] [PubMed] 